\title{
Dispersion of Electromagnetic Excitations in a Non-Ideal Microporous Lattice
}

\author{
Rumyantsev VV ${ }^{1,2 *}$ and Fedorov $\mathrm{SA}^{1}$ \\ ${ }^{1}$ Galkin Institute for Physics \& Engineering, Ukraine \\ ${ }^{2}$ Mediterranean Institute of Fundamental Physics, Rome, Italy
}

*Corresponding author: Rumyantsev VV, Galkin Institute for Physics \& Engineering, 83114

Donetsk, Ukraine, Email: vladimir.rumyantsev2011@yandex.ru

\section{Research Article}

Volume 5 Issue 2

Received Date: July 06, 2021

Published Date: July 21, 2021

DOI: $10.23880 / p s b j-16000182$

\section{Abstract}

We develop a numerical model for a defect-containing 2D lattice of microporous lattice with embedded ultracold atomic clusters (quantum dots). It is assumed that certain fractions of quantum dots and micropores are absent, which leads to transformation of polariton spectrum of the structure. The dispersion relations for polaritonic modes are derived as functions of defect concentrations and on this basis the band gap as well as the effective masses of lower and upper dispersion branch polaritons.

Keywords: Microporous lattice; Quantum dots; Polaritons; Band gap; Effective mass

\section{Introduction}

Fabrication and utilization of novel materials as sources of coherent irradiation constitute presently a vast interdisciplinary scientific area of experimental and theoretical research. It spans condensed matter physics, laser physics, nanotechnology, chemistry and information science. Particular actuality is gained by the investigations into possibilities of alteration of physical properties of the resulting composite structures by application of suitable external actions and perspectives of controlling of electromagnetic wave propagation therein. The existing optoelectronic devices can be roughly deviled into two types, namely those utilizing the disturbances of electronic subsystems of constituting materials, and those based on optical properties of the corresponding composite structures. However, lately the fabrication of optoelectronic devices gave rise to a new class of problems connected with formation of polaritonic structures [1,2] - a new type of photonic crystals [3] featured by a strong coupling between quantum disturbances of the medium (excitons) and electromagnetic field. The growing necessity for examination of polaritonic structures gave rise to polaritonics as an independent branch of photonics. The dissemination of optoelectronic devices creates an increasing interest for optical modes in microporous systems $[4,5]$ comprised by arrays of tunnelcoupled micropores (microresonators) and spatially periodic weakly interacting ensembles of two-level atoms. Such structures possess the property of polariton localization analogous to localization of light in photon crystals studied by non-linear optics (see Lee RK, Vučković J [6,7]) and exciton localization in quasi-periodic solid structures $[8,9]$. Lee RK [6] is devoted to defect-based resonators in photonic crystals; Amo A, et al. [10] contains demonstration of the attainment of strong binding between a quantum dot and such a resonator. In $[3,4]$ the formation of quantum solitons coupled to the lower-dispersion branch (LDB) polaritons was theoretically investigated for a chain of micropores. The authors Alodjants AP, Sedov ES, et al. [1,2] suggest that their result may prove useful for the purposes of quantum information processing. Microporous systems can also be employed for the construction of highly accurate optical 
clockworks [11-13]. Nanostructures such as microporous silicon [14], expected to solve the tasks of improvement of the functional complexity of the semiconductor devices.

Basing on the concepts of ideal polaritonic structures [3] and on the previously developed theory of exciton-like electromagnetic excitations [15] in Rumyantsev VV [16] we consider a non-ideal polaritonic crystal as a system of coupled micropores (microresonators). It is of interest to consider an array of such resonators with embedded atomic clusters (quantum dots). In the present article we study the dispersion of electromagnetic excitations in a non-ideal twodimensional lattice of coupled micropores as affected by the variable composition of quantum dots as well as the variable composition of the micropores. It is worthwhile recalling that the polariton model $[1,2,15,16]$ of the atomic-optical interaction utilized in this paper is valid only for the case of ultra-cold atoms with "frozen" (within the micropore) spatial degrees of freedom. This approximation holds for relatively small numbers of atoms embedded within each micropore $\left(N \leq 10^{4}\right.$ ) [17]. Parameter $g$ of the strong atomic-optical interaction should obey the relation

$$
g \gg 2 \pi / \tau_{c o h},(1)
$$

i.e. in each micropore $g$ should much exceed the inverse coherence time of the atomic-optical system [18]. Physically, $\tau_{c o h}$ is the time of thermodynamic equilibration of the atomic system, which interacts with electromagnetic field within the structure of polariton crystal. Inequality (1) is attained at low temperatures of the order of several $\mathrm{mK}$ when it is possible to neglect the spectral line broadening.

\section{Numerical Model of a Microporous Lattice}

A frequently used method to fabricate a polaritonic crystal is the trapping of two-level atoms in an ideal coupledresonator optical waveguide (CROW) [1,2] or in a nonideal array of microcavities $[15,16]$. To keep our discussion sufficiently general let us first of all consider a twodimensional microporous lattice with an arbitrary number $\sigma$ of sublattices and then tackle the important particular case of $\sigma=1$. Assume that the $\alpha$-th sublattice is composed of $s(\alpha)$ types of randomly distributed resonators, each of whom contains a quantum dot (a one-level atomic cluster) pertaining to one of $r(\alpha)$ types. Quantum dots interact with resonator-localized quantized electromagnetic fields and each of tunnel-coupled resonators possesses a single optical mode. In the coordinate representation the Hamiltonian of the described superstructure writes:

$$
\hat{H}=\hat{H}_{a t}+\hat{H}_{p h}+\hat{H}_{i n t}
$$

In (2) Hamiltonians of the atomic (quantum dot) subsystem $\hat{H}_{a t}$, photonic (micropores) subsystem $\hat{H}_{p h}$ and their interaction $\hat{H}_{i n t}$ equal correspondingly to

$\hat{H}_{a t}=\sum_{n} \hat{H}_{a t, n}+\frac{1}{2} \sum_{n, m} \hat{V}_{n m}{ }^{\prime} \quad \hat{H}_{p h}=\sum_{n} \hat{H}_{p h, n}-\frac{1}{2} \sum_{n, m} \hat{A}_{n m}$ $\hat{H}_{\text {int }}=\sum_{n} \hat{G}_{n}$, where $\hat{H}_{a t, n}$ is the Hamiltonian of a

stationary (ultracold) quantum dot embedded at the $n$-th micropore, $\hat{V}_{n m}$ is the operator of Coulomb interaction between quantum dots in $n$-th and $m$-th micropores, $\hat{H}_{p h, n}$ defines the state of electromagnetic excitation localized at the $n$-th micropore, $A_{n m}$ describes an overlap of optical fields of the $n$-th and $m$-th micropores (and hence the transfer probability of the corresponding electromagnetic excitation). Writing interaction operator $\hat{H}_{i n t}$ as a sum of unary operators $G_{n}$ is justified under the assumption that each of micropore-localized electromagnetic excitations interacts only with the quantum dot embedded at the same micropore. $n$ and $m$ are complex indices defined by the expressions $n \equiv(\mathbf{n}, \alpha), \quad m \equiv(\mathbf{m}, \beta)$, where twodimensional vectors $\mathbf{n}$ and $\mathbf{m}$ define positions of elementary cells in the superlattice, whereas $\alpha$ and $\beta$ numerate sublattices and assume values $1,2,3 \ldots \sigma$.

Let us in accordance with $[17,18]$ calculate the energy spectrum of the studied system by writing down its Hamiltonian $\hat{H}$ as a sum of the following second quantization

operators:

$$
\begin{aligned}
& \hat{H}_{a t}=\sum_{n, f} \varepsilon_{n f}^{a t} \hat{b}_{n f}^{+} \hat{b}_{n f}+\frac{1}{2} \sum_{n \neq m} \sum_{f, g} \sum_{h, l} \hat{b}_{n f}^{+} \hat{b}_{m g}^{+}\left\langle\varphi_{n f}^{a t} \varphi_{m g}^{a t}\left|V_{n m}\right| \varphi_{m h}^{a t} \varphi_{n l}^{a t}\right\rangle \hat{b}_{m h} \hat{b}_{n l}, \\
& \hat{H}_{p h}=\sum_{n, \mu} \varepsilon_{n \mu}^{p h} \hat{\phi}_{n \mu}^{+} \hat{\phi}_{n \mu}-\frac{1}{2} \sum_{n \neq m} \sum_{\mu, v} \sum_{\lambda, \rho} \hat{\phi}_{n \mu}^{+} \hat{\phi}_{m v}^{+}\left\langle\varphi_{n \mu}^{p h} \varphi_{m v}^{p h}\left|A_{n m}\right| \varphi_{m \lambda}^{p h} \varphi_{n \rho}^{p h}\right\rangle \hat{\phi}_{m \lambda} \hat{\phi}_{n \rho}, \\
& \hat{H}_{i n t}=\sum_{n, f, g} \sum_{\mu, v} \hat{b}_{n g}^{+} \phi_{n \mu}^{+}\left\langle\varphi_{n g}^{a t} \varphi_{n \mu}^{p h}\left|\hat{G}_{n}\right| \varphi_{n f}^{a t} \varphi_{n v}^{p h}\right\rangle \hat{b}_{n f} \hat{\phi}_{n v} .
\end{aligned}
$$

Here $\varepsilon_{n f}^{a t}, \varepsilon_{n \mu}^{p h}$ are the eigenvalues of operators $\hat{H}_{a t}$ and $\hat{H}_{p h}$, correspondingly. Wave functions $\varphi_{n f}^{a t}, \varphi_{n \lambda}^{p h}$ characterize the states of quantum dot and electromagnetic field at the $n$ -th resonator (micropore), while the Hermitian conjugate (non-Hermitian) creation and annihilation operators $\hat{b}_{n f}^{+}$, $\hat{b}_{n f}, \hat{\phi}_{n \mu}^{+}, \hat{\phi}_{n \mu}$ describe, correspondingly, the states $f$ of 
quantum dot and the states $\mu$ of electromagnetic field at the $n$-th micropore. $\hat{b}_{n f}^{+}, \hat{b}_{n f}$ and $\hat{\phi}_{n \mu}^{+}, \hat{\phi}_{n \mu}$ are Pauli operators, which satisfy the following commutation relations $\hat{b}_{n f} \hat{b}_{m g}^{+}+\hat{b}_{m g}^{+} \hat{b}_{n f}=1, \hat{b}_{n f} \hat{b}_{m g}=\hat{b}_{n f}^{+} \hat{b}_{m g}^{+}=0$ for $n f=m g$ and $\hat{b}_{n f} \hat{b}_{m g}-\hat{b}_{m g} \hat{b}_{n f}=\hat{b}_{n f} \hat{b}_{m g}^{+}-\hat{b}_{m g}^{+} \hat{b}_{n f}=0$ for $\quad n f \neq m g$ (analogous commutation relations hold for $\hat{\phi}_{n \mu}^{+}$and $\hat{\phi}_{n \mu}$ ). The determination of the wave functions of the quantum dots $\varphi_{n f}^{a t}$ and the electromagnetic excitations localized in the micropores $\varphi_{n \lambda}^{p h}$ (and also the corresponding energies $\varepsilon_{n f}^{a t}$, $\varepsilon_{n \mu}^{p h}$ ) is achieved by means of a self-consistent variational procedure, as described in Davydov S [18].

Let us make a reasonable assumption that the densities of excited states of elements in both constituent subsystems (atomic and resonator) is a small quantity. This allows to simplify the energy operator (2)-(3) by approximating Pauli operators with Bose operators $\hat{b}_{n f}^{+} \hat{b}_{n 0} \approx \hat{B}_{n f}^{+}, \hat{b}_{n 0}^{+} \hat{b}_{n f} \approx \hat{B}_{n f}$,

$\hat{\phi}_{n \mu}^{+} \hat{\phi}_{n 0} \approx \hat{\Psi}_{n \mu}^{+}, \quad \hat{\phi}_{n 0}^{+} \hat{\phi}_{n \mu} \approx \hat{\Psi}_{n \mu} . \quad$ Next, since we are constructing a one-level model, indices $f, g, h, l$ in (3) should assume the values 0 and $a$, while indices $\mu, v, \lambda$, $\rho$ assume values 0 and 1 . Therefore within the HeitlerLondon approximation the quadratic (in $\hat{B}_{n f}$ and $\hat{\Psi}_{n \mu}$ ) part of Hamiltonian (3) is given by the following expressions:

$$
\begin{aligned}
& \hat{H}_{a t}^{H-L}=\sum_{n}\left(\Delta \varepsilon_{n a}+\sum_{m}\left(\left\langle\varphi_{n a}^{a t} \varphi_{m 0}^{a t}\left|\hat{V}_{n m}\right| \varphi_{m 0}^{a t} \varphi_{n a}^{a t}\right\rangle-\left\langle\varphi_{n 0}^{a t} \varphi_{m 0}^{a t}\left|\hat{V}_{n m}\right| \varphi_{m 0}^{a t} \varphi_{n 0}^{a t}\right\rangle\right)\right) \hat{B}_{n a}^{+} \hat{B}_{n a}+ \\
& +\sum_{n, m}\left\langle\varphi_{n 0}^{a t} \varphi_{m a}^{a t}\left|\hat{V}_{n m}\right| \varphi_{m 0}^{a t} \varphi_{n a}^{a t}\right\rangle \hat{B}_{n a}^{+} \hat{B}_{m a} \equiv \sum_{n} \hbar \omega_{n a}^{a t} \hat{B}_{n a}^{+} \hat{B}_{n a}+\sum_{n, m} V_{n m}^{(a)} \hat{B}_{n a}^{+} \hat{B}_{m a},
\end{aligned}
$$

$$
\begin{aligned}
& \hat{H}_{i n t}^{H-L}=\sum_{n} \hat{\Psi}_{n 1}^{+} \hat{B}_{n a}\left\langle\varphi_{n 0}^{a t} \varphi_{n 1}^{p h}\left|\hat{G}_{n}\right| \varphi_{n a}^{a t} \varphi_{n 0}^{p h}\right\rangle+\hat{\Psi}_{n 1} \hat{B}_{n a}^{+}\left\langle\varphi_{n a}^{a t} \varphi_{n 0}^{p h}\left|\hat{G}_{n}\right| \varphi_{n 0}^{a t} \varphi_{n 1}^{p h}\right\rangle \equiv \\
& \equiv \sum_{n} g_{n}\left(\hat{\Psi}_{n 1}^{+} \hat{B}_{n a}+\hat{\Psi}_{n 1} \hat{B}_{n a}^{+}\right) .
\end{aligned}
$$

$$
\begin{aligned}
& \hat{H}_{p h}^{H-L}=\sum_{n}\left(E_{n 1}-\sum_{m}\left(\left\langle\varphi_{n 1}^{p h} \varphi_{m 0}^{p h}\left|\hat{A}_{n m}\right| \varphi_{m 0}^{p h} \varphi_{n 1}^{p h}\right\rangle-\left\langle\varphi_{n 0}^{p h} \varphi_{m 0}^{p h}\left|\hat{A}_{n m}\right| \varphi_{m 0}^{p h} \varphi_{n 0}^{p h}\right\rangle\right)\right) \hat{\Psi}_{n 1}^{+} \hat{\Psi}_{n 1}- \\
& -\sum_{n, m}\left\langle\varphi_{n 0}^{p h} \varphi_{m 1}^{p h}\left|\hat{A}_{n m}\right| \varphi_{m 0}^{p h} \varphi_{n 1}^{p h}\right\rangle \hat{\Psi}_{n 1}^{+} \hat{\Psi}_{m 1} \equiv \sum_{n} \hbar \omega_{n 1}^{p h} \hat{\Psi}_{n 1}^{+} \hat{\Psi}_{n 1}-\sum_{n, m} A_{n m} \hat{\Psi}_{n 1}^{+} \hat{\Psi}_{m 1}
\end{aligned}
$$

In (4) and (5) appear the frequency characteristics $\omega_{n 1}^{p h}$ , $\omega_{n \alpha}^{a t}$ of micropore (resonator) and atomic subsystems as well as the matrix of resonant coupling:

$$
\begin{gathered}
\left\langle\varphi_{n 0}^{a t} \varphi_{m a}^{a t}\left|\hat{V}_{n m}\right| \varphi_{m 0}^{a t} \varphi_{n a}^{a t}\right\rangle \equiv V_{n m}^{(a)}, \\
\left\langle\varphi_{n 0}^{p h} \varphi_{m 1}^{p h}\left|\hat{A}_{n m}\right| \varphi_{m 0}^{p h} \varphi_{n 1}^{p h}\right\rangle \equiv A_{n m} .
\end{gathered}
$$

In (6) it is taken into account that the wave functions of quantum dots and electromagnetic fields are real-valued and hence $\left\langle\varphi_{n 0}^{a t} \varphi_{n 1}^{p h}\left|\hat{G}_{n}\right| \varphi_{n a}^{a t} \varphi_{n 0}^{p h}\right\rangle=\left\langle\varphi_{n a}^{a t} \varphi_{n 0}^{p h}\left|\hat{G}_{n}\right| \varphi_{n 0}^{a t} \varphi_{n 1}^{p h}\right\rangle \equiv g_{n}$.

The deviation from ideality of the considered system consists in disordering of its resonant and atomic subsystems. Mathematically this is reflected in the fact that the quantities $\omega_{n 1}^{p h}, \omega_{n a}^{a t}, V_{n m}^{(a)}, A_{n m}$ and $g_{n}$ are configurationally dependent and therefore Hamiltonian (2) is not translation invariant. One of the methods of obtaining the spectra of quasiparticle excitations in disordered systems with randomly distributed elements consists in finding the poles of configurationally averaged resolvent of the appropriate Hamiltonian [19]. The said resolvent is translation invariant and so the corresponding elementary excitation spectrum can be described by a wave vector $\mathbf{k}$. To carry out the necessary calculation one should inevitably adopt a certain approximation, whose choice is dictated by the specifics of the studied system. A widespread tool for evaluation of quasiparticle states in disordered media is the virtual crystal approximation (VCA) $[19,20]$. It is particularly suitable for tracing the effect of defect concentrations on the specifics of the spectrum and the related quantities. Within the VCA the averaged resolvent equals to the resolvent of the averaged Hamiltonian, and it is the latter, which needs to be diagonalized in order to calculate the spectrum. In what follows we use this approximation to analyze electromagnetic excitations and optical characteristics of the studied microporous superstructure.

Let us, by analogy with $[15,16]$ express the configurationally dependent quantities $\omega_{n a}^{a t} \equiv \omega_{\mathbf{n} \alpha}^{a t}$, $\omega_{n 1}^{p h} \equiv \omega_{\mathbf{n} \alpha}^{p h}, \quad V_{n m}^{(a)}, A_{n m} \quad$ and $g_{n}$ through the random quantities $\eta_{a t, n \alpha}^{\nu(\alpha)}\left(\eta_{p h, n \alpha}^{\nu(\alpha)}\right)$ :

$$
\begin{aligned}
& \omega_{\mathbf{n} \alpha}^{a t}=\sum_{v(\alpha)=1}^{r(\alpha)} \omega_{a t, \alpha}^{v(\alpha)} \eta_{a t, \mathbf{n} \alpha}^{v(\alpha)} ; \quad V_{\mathbf{n} \alpha \mathbf{m} \beta}^{(a)}=\sum_{v(\alpha), \mu(\beta)=1}^{r(\alpha) r(\beta)} V_{\alpha \beta}^{v(\alpha) \mu(\beta)}(\mathbf{n}-\mathbf{m}) \eta_{a t, \mathbf{n} \alpha}^{v(\alpha)} \eta_{a t, \mathbf{m} \beta}^{\mu(\beta)}, \\
& \omega_{\mathbf{n} \alpha}^{p h}=\sum_{v(\alpha)=1}^{s(\alpha)} \omega_{p h, \alpha}^{v(\alpha)} \eta_{p h, \mathbf{n} \alpha}^{v(\alpha)} ; \quad A_{\mathbf{n} \alpha \mathbf{m} \beta}=\sum_{v(\alpha), \mu(\beta)=1}^{s(\alpha) s(\beta)} A_{\alpha \beta}^{v(\alpha) \mu(\beta)}(\mathbf{n}-\mathbf{m}) \eta_{p h, \mathbf{n} \alpha}^{v(\alpha)} \eta_{p h, \mathbf{m} \beta}^{\mu(\beta)} .
\end{aligned}
$$


Configurational dependence of both constituent subsystems is reflected in the quantity $g_{n}$

$$
g_{n}=\sum_{v(\alpha)=1}^{r(\alpha)} \sum_{\mu(\alpha)=1}^{s(\alpha)} g_{\alpha}^{v(\alpha) \mu(\alpha)} \eta_{a t, \mathbf{n} \alpha}^{v(\alpha)} \eta_{p h, \mathbf{n} \alpha}^{\mu(\alpha)}
$$

where $\eta_{a t, \mathbf{n} \alpha}^{v(\alpha)}=1\left(\eta_{p h, \mathbf{n} \alpha}^{v(\alpha)}=1\right)$ if the $\mathbf{n} \alpha$-th site is occupied by a quantum dot (resonator) of the $v(\alpha)$-th type and $\eta_{a t, \mathbf{n} \alpha}^{v(\alpha)}=0\left(\eta_{p h, \mathbf{n} \alpha}^{v(\alpha)}=0\right)$ in all other cases. On the assumption that the ordering states of the two subsystems are independent of each other we arrive at the following expressions for the configurationally averaged quantities

$$
\begin{aligned}
& \left\langle\omega_{\mathbf{n} \alpha}^{a t}\right\rangle=\sum_{v(\alpha)=1}^{r(\alpha)} \omega_{a t, \alpha}^{v(\alpha)} C_{a t, \alpha}^{v(\alpha)} ;\left\langle V_{\mathbf{n} \alpha \mathbf{m} \beta}^{(a)}\right\rangle=\sum_{v(\alpha), \mu(\beta)=1}^{r(\alpha) r(\beta)} V_{\alpha \beta}^{v(\alpha) \mu(\beta)}(\mathbf{n}-\mathbf{m}) C_{a t, \alpha}^{v(\alpha)} C_{a t, \beta}^{\mu(\beta)}, \\
& \left\langle\omega_{\mathbf{n} \alpha}^{p h}\right\rangle=\sum_{v(\alpha)=1}^{s(\alpha)} \omega_{p h, \alpha}^{v(\alpha)} C_{p h, \alpha}^{v(\alpha)} ;\left\langle A_{\mathbf{n} \alpha \mathbf{m} \beta}\right\rangle=\sum_{v(\alpha), \mu(\beta)=1}^{s(\alpha) s(\beta)} A_{\alpha \beta}^{v(\alpha) \mu(\beta)}(\mathbf{n}-\mathbf{m}) C_{p h, \alpha}^{v(\alpha)} C_{p h, \beta}^{\mu(\beta)}, \\
& \left\langle g_{n}\right\rangle=\sum_{\nu(\alpha)=1}^{r(\alpha)} \sum_{\mu(\alpha)=1}^{s(\alpha)} g_{\alpha}^{v(\alpha) \mu(\alpha)} C_{a t, \alpha}^{v(\alpha)} C_{p h, \alpha}^{\mu(\alpha)},
\end{aligned}
$$

where angular brackets denote the averaging procedure. $C_{a t, \alpha}^{\nu(\alpha)}\left(C_{p h, \alpha}^{\nu(\alpha)}\right)$ and $C_{a t, \beta}^{\mu(\beta)}\left(C_{p h, \beta}^{\mu(\beta)}\right)$ denote concentrations of

the $v(\alpha)$-th and $\mu(\beta)$-th type of elements of the atomic or resonator subsystems. There hold the obvious relations $\sum_{v(\alpha)}^{r(\alpha)} C_{a t, \alpha}^{v(\alpha)}=1 \quad \sum_{v(\alpha)}^{s(\alpha)} C_{p h, \alpha}^{v(\alpha)}=1$

Configurational averaging allows to "restore" the translation invariance of a nonideal superstructure (which is essentially the central idea of the VCA) and to characterize the eigenvalues and eigenfunctions of Hamiltonian $\langle H\rangle$ of the resulting virtual crystal by a wave vector $\mathbf{k}=\left(k_{x}, k_{y}, 0\right)$ . In $\mathbf{k}$-representation Hamiltonian $\langle H\rangle$ writes as

$$
\langle\hat{H}\rangle_{\mathbf{k}}=\left\langle\hat{H}_{a t}\right\rangle_{\mathbf{k}}+\left\langle\hat{H}_{p h}\right\rangle_{\mathbf{k}}+\left\langle\hat{H}_{i n t}\right\rangle_{\mathbf{k}}
$$

where

$$
\begin{aligned}
& \left\langle\hat{H}_{a t}\right\rangle_{\mathbf{k}}=\sum_{\alpha, \beta}\left[\hbar\left\langle\omega_{\mathbf{n} \alpha}^{a t}\right\rangle \delta_{\alpha \beta}+V_{\alpha \beta}^{(a)}(\mathbf{k})\right] \hat{B}_{\alpha a}^{+}(\mathbf{k}) \hat{B}_{\beta a}(\mathbf{k}), \\
& \left\langle\hat{H}_{p h}\right\rangle_{\mathbf{k}}=\sum_{\alpha, \beta}\left[\hbar\left\langle\omega_{\mathbf{n} \alpha}^{p h}\right\rangle \delta_{\alpha \beta}-A_{\alpha \beta}(\mathbf{k})\right] \hat{\Psi}_{\alpha 1}^{+}(\mathbf{k}) \hat{\Psi}_{\beta 1}(\mathbf{k}), \\
& \left\langle\hat{H}_{i n t}\right\rangle_{\mathbf{k}}=\sum_{\alpha}\left\langle g_{\mathbf{n} \alpha}\right\rangle\left[\hat{\Psi}_{\alpha 1}^{+}(\mathbf{k}) \hat{B}_{\alpha a}(\mathbf{k})+\hat{\Psi}_{\alpha 1}(\mathbf{k}) \hat{B}_{\alpha a}^{+}(\mathbf{k})\right] .
\end{aligned}
$$

Here $V_{\alpha \beta}^{(a)}(\mathbf{k}), A_{\alpha \beta}(\mathbf{k}), \hat{B}_{\alpha a}(\mathbf{k}), \hat{\Psi}_{\alpha 1}(\mathbf{k})$ are the Fourier components of $\left\langle V_{\mathbf{n} \alpha \mathbf{m} \beta}^{(a)}\right\rangle,\left\langle A_{\mathbf{n} \alpha \mathbf{m} \beta}\right\rangle, \quad \hat{B}_{\mathbf{n} a}$ and $\quad \hat{\Psi}_{n 1}$, respectively $([15,16])$.

Diagonalization of Hamiltonian $\langle\hat{H}\rangle_{\mathbf{k}}$ by the use of Bogolyubov-Tyablikov transformation [17] yields the expressions for the energies of polariton excitations in the considered microporous crystal with embedded quantum dots.

\section{Results and Discussion}

To make our further discussion more specific let us concentrate on polariton excitations in a defect-containing one-sublattice square Bravais lattice with period $d$ (Figure

1). The role of defects shall be played by vacancies contained in both atomic and micropore subsystems. In such a case $C_{a t, 1}^{(1)} \equiv C_{1}^{V}$ and $C_{p h, 1}^{(1)} \equiv C_{2}^{V}$. Simplicity of the structure permits to reduce the somewhat cumbersome notations of the previous section to a more comprehensible form. Namely, we shall operate with the quantities $V_{11} \equiv V, A_{11} \equiv A$, $g_{1}^{11} \equiv g, \omega_{p h, 1}^{1} \equiv \omega_{p h}, \omega_{a t, 1}^{1} \equiv \omega_{a t}$. The above mentioned diagonalization procedure of Hamiltonian $\langle H\rangle_{\mathbf{k}}$ leads to a system of linear homogeneous equations, whose solvability condition is formulated as the equality of the following determinant to zero:

$$
\left\|\begin{array}{cc}
\hbar \omega^{a t}(\mathbf{k})-\hbar \Omega(\mathbf{k}) & \left\langle g_{\mathbf{n}}\right\rangle \\
\left\langle g_{\mathbf{n}}\right\rangle & \hbar \omega^{p h}(\mathbf{k})-\hbar \Omega(\mathbf{k})
\end{array}\right\|=0
$$




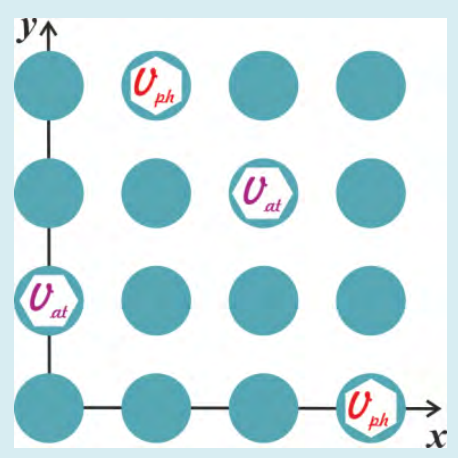

Figure 1: Schematic of a modeling square one-sublattice array of micropores. Solid circles denote defect-free cavities with embedded quantum dots. $V_{a t}$-type of defect is a cavity with a missing quantum dot. $V_{p h}$-type of defect

is an empty site with no micropore (and hence with no quantum dot, since atomic clusters can only reside at the existent micropores). Defect concentration in the atomic subsystem (i.e. concentration of sites with missing quantum dots) $C_{1}^{V}$ equals to the sum of concentrations of the $V_{a t}$ - and $V_{p h}$-types of defects. Defect concentration in the photonic subsystem (concentration of sites with missing micropores) $C_{2}^{V}$ equals to the $V_{p h}$-type defect concentration. There holds an obvious inequality $C_{1}^{V} \geqslant$ $C_{2}^{V}$.

Here the energies of exciton exitations of the atomic (quantum dot) subsystem and of exciton-like electromagnetic exitations [15] of the resonator (micropore) subsystem within the nearest-neighbor approximation equal correspondingly to:

$$
\begin{aligned}
& \hbar \omega^{a t}(\mathbf{k})=\hbar\left\langle\omega_{\mathbf{n}}^{a t}\right\rangle+2 V(d)\left(1-C_{1}^{V}\right)^{2}\left(\cos k_{x} d+\cos k_{y} d\right), \\
& \hbar \omega^{p h}(\mathbf{k})=\hbar\left\langle\omega_{\mathbf{n}}^{p h}\right\rangle-2 A(d)\left(1-C_{2}^{V}\right)^{2}\left(\cos k_{x} d+\cos k_{y} d\right) .
\end{aligned}
$$

Equality (13) defines a quadratic equation for the unknown dispersion dependence $\Omega(\mathbf{k})$. According to (10) polariton frequencies $\Omega(\mathbf{k})$ along with parameters $\left\langle\omega_{\mathbf{n}}^{a t}\right\rangle,\left\langle\omega_{\mathbf{n}}^{p h}\right\rangle$, $V(\mathbf{k}), A(\mathbf{k})$ and $\left\langle g_{\mathbf{n}}\right\rangle$ are the functions of structural elements in the two subsystems (quantum dots and resonators). In the considered case in view of (9) we obtain $\left\langle\omega_{\mathbf{n}}^{a t}\right\rangle=\left(1-C_{1}^{V}\right) \omega_{a t}$,

$$
\left\langle\omega_{\mathbf{n}}^{p h}\right\rangle=\left(1-C_{2}^{V}\right) \omega_{p h}{ }^{\prime}
$$

$\left\langle g_{\mathbf{n}}\right\rangle=g\left(1-C_{1}^{V}\right)\left(1-C_{2}^{V}\right) \cdot C_{1}^{V} \quad$ and $\quad C_{2}^{V}$ denote concentrations of defects (vacancies) in the atomic and resonator subsystems respectively. The transfer probability of electromagnetic excitation between the nearest neighbor sites is defined by an overlap characteristic of optical fields $A(d) \cdot V(d)$ describes the Coulomb interaction between quantum dots in neighboring micropores.

Substitution of expressions (14) for $\hbar \omega^{a t}(\mathbf{k})$ and $\hbar \omega^{p h}(\mathbf{k})$ into (13) yields the dispersion law $\Omega_{ \pm}\left(\mathbf{k}, C_{1}^{V}, C_{2}^{V}\right)$ of electromagnetic excitations in the micropores array (where plus and minus signs stand for the upper and lower dispersion bands, respectively). We have performed the numerical evaluation of $\Omega_{ \pm}\left(\mathbf{k}, C_{1}^{V}, C_{2}^{V}\right)$ for several permissible concentration values falling within the domain $C_{1}^{V} \geqslant C_{2}^{V}$ (defined by inequality, which accounts for

the fact that quantum dots can only reside in existent micropores). Parameters, which describe an interaction between the atomic and photonic subsystems as well as an overlap of optical fields and an interaction between quantum dots in neighboring micropores were set equal correspondingly to $g / \hbar=7 \cdot 10^{13} \mathrm{~Hz}$, $A(d) / 2 \hbar=3.5 \cdot 10^{14} \mathrm{~Hz}, \quad V(d) / 2 \hbar=9 \cdot 10^{13} \mathrm{~Hz} ; \quad$ the lattice period was taken to be $d=3 \cdot 10^{-7} \mathrm{~m}$. In Figure 2a the dispersion bands $\Omega_{ \pm}\left(k_{x}, k_{y}, C_{1}^{V}, C_{2}^{V}\right)$ are plotted for an ideal structure $\left(C_{1}^{V}=C_{2}^{V}=0\right)$, Figure 2b illustrates their transformation under the decrease of parameter $g$ (responsible for interaction between the atomic and photonic subsystems) by a factor of 10 . Figure $2 \mathrm{c}$ gives an example of dispersion bands of a nonideal structure $\left(C_{1}^{V}=0.43\right.$,

$C_{2}^{V}=0.2$ ).It should be noted that the shape of the dispersion

curve in Fig. 2a,b indicates the existence of Bose-Einstein exciton condensate, where the energy minima occur for a number of states with non-zero $k$ 's (in addition to those with $k=0$ ). In Figure 3 is shown the concentration

dependence of the band gap width

$$
\Delta \Omega\left(C_{1}^{V}, C_{2}^{V}\right) \equiv \min _{\mathbf{k}} \Omega_{+}\left(\mathbf{k}, C_{1}^{V}, C_{2}^{V}\right)-\max _{\mathbf{k}} \Omega_{-}\left(\mathbf{k}, C_{1}^{V}, C_{2}^{V}\right) .
$$




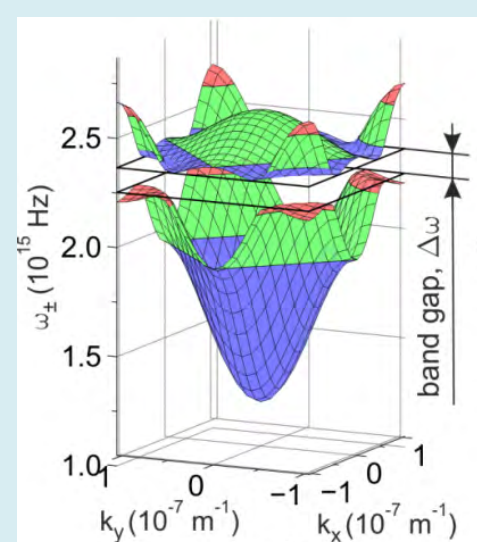

a)

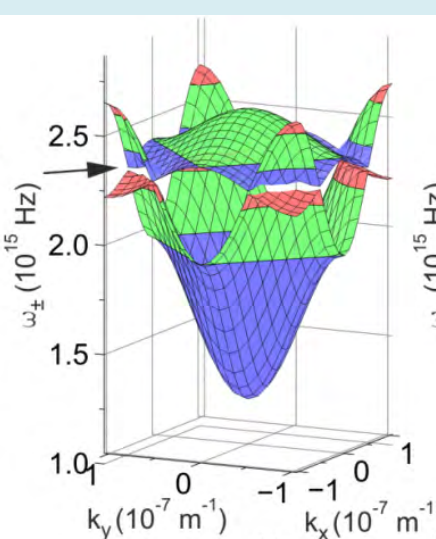

b)

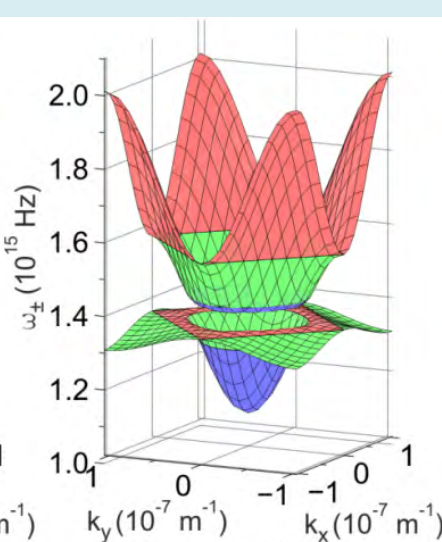

c)

Figure 2: Polariton energy bands of an ideal (a, b) micropores array $\left(C_{1}^{V}=C_{2}^{V}=0\right)$ and a defect-containing (c) array ( $C_{1}^{V}=0.43, C_{2}^{V}=0.2$ ). (b) illustrates the narrowed "bottle neck" resulting from the decrease of parameter $g$ (responsible

for interaction between the atomic and photonic subsystems) by a factor of 10 .

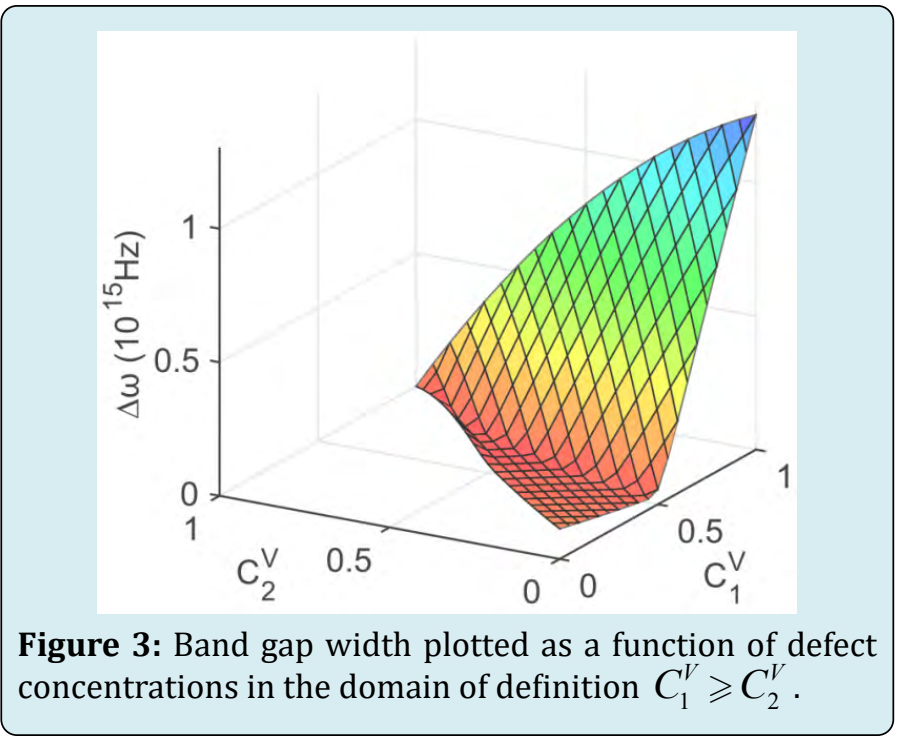

An important property of band gap photonic materials is theirabilitytoproducetheso-called "slow"light, which appears to be highly promising for the purposes of construction and utilization of quantum information processing devices [21]. An efficient reduction of quasiparticle group velocity was demonstrated e.g. in coupled-resonator optical waveguides $[22,23]$ as well as in solid-state multilayer semiconductor structures [24]. The theoretical study [25] of the photonic band structure of binary one-dimensional arrays of tunnelcoupled microcavities shows that subjecting the system to the controllable elastic strain is an effective tool for altering its eigen mode structure and optical properties.

The key role in the reduction of the said group velocity is played by the character of the effective masses $m_{\text {eff }}^{( \pm)}$of the so-called "dark" and "bright" polaritons, which arise in the specified materials as linear superpositions of the photonic states of resonator subsystems and the coherent excitations of one-level atomic subsystems. Concentration dependencies of the effective masses of upper and lower dispersion branch polaritons are given by the formula:

$$
m_{e f f}^{( \pm)}\left(C_{1}^{V}, C_{2}^{V}\right) \equiv \hbar\left(\left.\frac{\partial^{2} \Omega_{ \pm}\left(\mathbf{k}, C_{1}^{V}, C_{2}^{V}\right)}{\partial k_{x}^{2}}\right|_{\substack{k_{x}=0 \\ k_{y}=0}}\right)^{-1}
$$

We have performed their numerical calculation; the results are shown in Figures $4 \mathrm{a} \& 4 \mathrm{~b}$. Examination of Figures 4a,b permits to conclude that an appropriate choice of defect concentrations $C_{1}^{V}, C_{2}^{V}$ may yield in principle the desirable characteristics of the "slow" light.

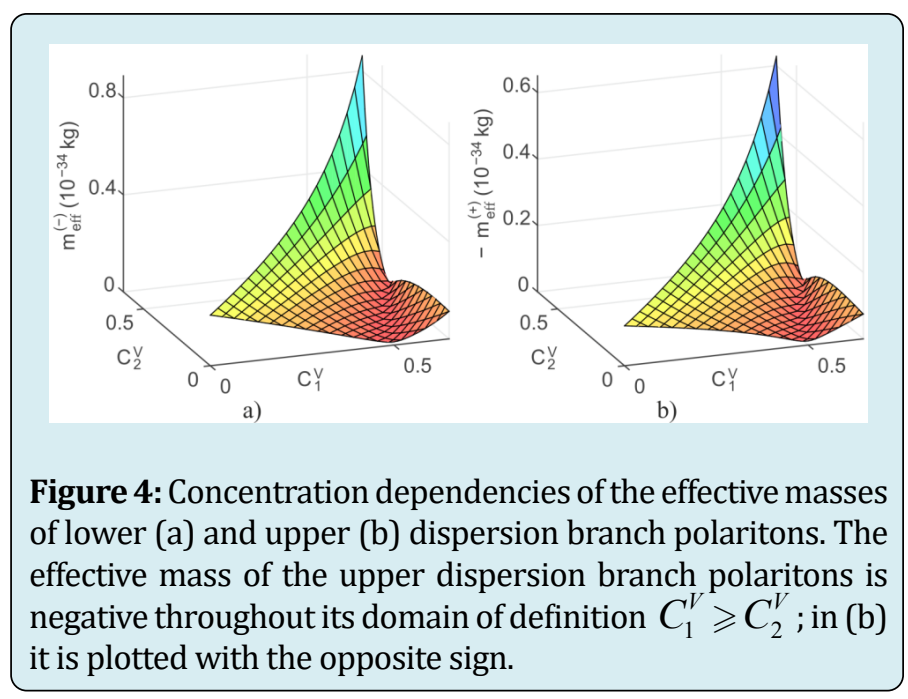




\section{Conclusion}

The paper is devoted to elucidation of the effect of pointlike defects on polariton dispersion in a two-dimensional microporous lattice with embedded one-level quantum dots. It is shown that the presence of vacancies in the resonator and atomic subsystems results in a substantial renormalization of polariton spectrum and thus in a considerable alteration of optical properties of the structure. Introduction of defects leads to an increase in the effective masses of polaritons and hence to a decrease of their group velocity. Our model is primarily based on the virtual crystal approximation, which is often employed to examine quasiparticle excitations in sufficiently simple disordered superstructures. More complex systems usually require the use of more sophisticated methods such as the (one- or multinode) coherent potential approximation [20], the averaged T-matrix method and their various modifications. The obtained numerical results contribute to our understanding of composite polaritonic structures and the prospects of their utilization for construction of solid-state devices with controllable propagation of electromagnetic waves.

\section{References}

1. Alodjants AP, Barinov IO, Arakelian SM (2010) Strongly localized polaritons in an array of trapped two-level atoms interacting with a light field. J Phys B At Mol Opt Phys 43: 095502.

2. Sedov ES, Alodjants AP, Arakelian SM, Chuang YL, Lin YY, et al. (2014) Tunneling-assisted optical information storage with lattice polariton solitons in cavity-QED arrays. Phys Rev A 89: 033828.

3. Joannopoulos JD, Johnso SG, Winn JN, Meade RD (2008) Photonic Crystals: Molding the Flow of Light. 2nd(Edn.), Princeton University Press, Princeton, USA.

4. Kaliteevskii MA (1997) Coupled vertical microcavities. Tech Phys Lett 23(2): 120-121.

5. Vahala KJ (2003) Optical microcavities. Nature 424: 839846.

6. Lee RK, Painter O, Kitzke B, Scherer A, Yariv A (2000) Emission properties of a defect cavity in a twodimensional photonic bandgap crystal slab. J Opt Soc Am B 17(4): 629-633.

7. Vučković J, Loncar M, Mabuchi H, Scherer A (2001) Design of photonic crystal microcavities for cavity QED. Phys Rev E Stat Nonlin Soft Matter Phys 65(1 Pt 2): 016608.

8. Balili R Hartwell V, Snoke D, Pfeiffer L, West K (2007)
Bose-Einstein condensation of microcavity polaritons in a trap. Science 316: 1007-10010.

9. Amo A, Lefrère J, Pigeon S, Adrados C, Ciuti C, et al. (2009) Superfluidity of polaritons in semiconductor microcavities. Nature Physics 5: 805-810.

10. Englund D, Majumdar A, Faraon A, Toish M, Stoltz N, et al. (2010) Resonant Excitation of a Quantum Dot Strongly Coupled to a Photonic Crystal Nanocavity. Phys Rev Lett 104(7): 073904.

11. Del'Haye PD, Schliesser A, Arcizet O, Wilken T, Holzwarth $\mathrm{R}$, et al. (2007) Optical frequency comb generation from a monolithic microresonator. Nature 450: 1214-1217.

12. Hou D, Ning B, Wu J, Wang Z, Zhao J (2013) Demonstration of a stable erbium-fiber-laser-based frequency comb based on a single rubidium atomic resonator. Appl Phys Lett 102: 151104.

13. Papp SB, Beha K, Del'Haye P, Quinlan F, Lee H, et al. (2014) Microresonator frequency comb optical clock. Optica 1(1): 10-14.

14. Zhao J, Luo G, Wu J, Xia H (2013) Preparation of Microporous Silicone Rubber Membrane with Tunable Pore Size via Solvent Evaporation-Induced Phase Separation. ACS Appl Mater Interface 5(6): 2040-2046.

15. Rumyantsev VV, Fedorov SA, Gumennyk KV, Sychanova MV, Kavokin AV (2014) Exciton-like electromagnetic excitations in non-ideal microcavity supercrystals. Nature 4: 6945.

16. Rumyantsev VV, Fedorov SA, Gumennyk KV, Sychanova MV (2015) Dispersion characteristics of electromagnetic excitations in a disordered 1D lattice of coupled microresonators. Physica B 461: 32-37.

17. Agranovich VM (1968) Theory of Excitons. Nauka Publishers, Moscow, Russia.

18. Davydov S (1962) Theory of molecular excitons. 1st(Edn.), McGraw Hill, New York, pp: 313.

19. Ziman JM (1979) Models of disorder: The theoretical physics of homogeneously disordered systems. Cambridge University Press, Cambridge, USA.

20. Los' VF (1987) Projection operator method in the theory of disordered systems. I. Spectra of quasiparticles. Theor Math Phys 73: 1076-1088.

21. Milonni PW (2005) Fast Light, Slow Light and LeftHanded Light. Institute of Physics Publishing, Bristol, pp: 262. 
Physical Science \& Biophysics Journal

22. Yang ZS, Kwong NH, Binder R, Smirl AL (2005) Stopping, storing, and releasing light in quantum-well Bragg structures. J Opt Soc Am B 22(10): 2144-2156.

23. Gersen H, Karle TJ, Engelen RJP, Bogaerts W, Korterik JP, et al. (2005) Real-Space Observation of Ultraslow Light in Photonic Crystal Waveguides. Phys Rev Lett 94: 073903.

24. Turukhin AV, Sudarshanam VS, Shahriar MS, Musser
JA, Ham BS, et al. (2002) Observation of Ultraslow and Stored Light Pulses in a Solid. Phys Rev Lett 88(2): 023602.

25. Rumyantsev VV, Fedorov SA, Gumennyk KV, Gurov DA, Kavokin AV (2018) Effects of elastic strain and structural defects on slow light modes in a one-dimensional array of microcavities. Superlattices and Microstructures 120: 642-649. 